\title{
Semi-empirical validation of the cross-band relative absorption technique for the measurement of molecular mixing ratios
}

\author{
Denis Pliutau, and Narasimha S. Prasad \\ NASA Langley Research Center, 5 N. Dryden St., MS 468, Hampton VA, 23681
}

\begin{abstract}
Studies were performed to carry out semi-empirical validation of a new measurement approach we propose for molecular mixing ratios determination. The approach is based on relative measurements in bands of $\mathrm{O}_{2}$ and other molecules and as such may be best described as cross band relative absorption (CoBRA). . The current validation studies rely upon well verified and established theoretical and experimental databases, satellite data assimilations and modeling codes such as HITRAN, line-by-line radiative transfer model (LBLRTM), and the modern-era retrospective analysis for research and applications (MERRA). The approach holds promise for atmospheric mixing ratio measurements of $\mathrm{CO}_{2}$ and a variety of other molecules currently under investigation for several future satellite lidar missions. One of the advantages of the method is a significant reduction of the temperature sensitivity uncertainties which is illustrated with application to the ASCENDS mission for the measurement of $\mathrm{CO}_{2}$ mixing ratios $\left(\mathrm{XCO}_{2}\right)$. Additional advantages of the method include the possibility to closely match cross-band weighting function combinations which is harder to achieve using conventional differential absorption techniques and the potential for additional corrections for water vapor and other interferences without using the data from numerical weather prediction (NWP) models.
\end{abstract}

Keyword list: lidar, molecular mixing ratio, HITRAN, spectroscopy, absorption, radiative transfer

\section{INTRODUCTION}

Several active sensing lidar missions relying on Integrated Path Differential Absorption (IPDA) approach for the mixing ratio measurements of the atmospheric molecules such as $\mathrm{CO}_{2}, \mathrm{CH}_{4}$ and $\mathrm{N}_{2} \mathrm{O}$ are being investigated. ${ }^{1-7}$ Examples of these future lidar missions include Active Sensing of $\mathrm{CO}_{2}$ Emissions Over Nights Days and Seasons (ASCENDS), Methane Remote Lidar Mission (MERLIN), and Advanced Space Carbon and Climate Observation of Planet Earth (A-SCOPE).$^{5-7}$ Previous studies have identified a number of spectral interferences such as those due to temperature, pressure variations and water vapor absorption as making significant contribution to the total estimated mixing ratio uncertainties. ${ }^{1-7}$ One of the ways these uncertainties due to variations in atmospheric parameters of temperature, pressure and humidity are carried over to the derived mixing ratios is through the extraction of the altitude distribution of the column averaged mixing ratio values by means of fitting techniques which utilize atmospheric profiles data only known with a limited precision. ${ }^{8}$ The uncertainties introduced this way could be significantly reduced if the need in the altitude mixing ratio fitting could be eliminated or the accuracy requirements on the known atmospheric data relaxed.

To address the accuracy limitations imposed by the temperature and pressure induced uncertainties in the IPDA technique and provide a way for additional optimizations to reduce the influence of water vapor interferences, we have suggested a Cross-Band Relative Absorption technique which relies on spectral lines matching in bands of various molecules (i.e. $\mathrm{CO}_{2}, \mathrm{CH}_{4}, \mathrm{~N}_{2} \mathrm{O}$ etc.) with those located in a reference band of oxygen such as that at 1.26-1.27 $\mu$ m. ${ }^{9}$ The 
key component of the proposed CoBRA methodology is the reduction of the temperature and total pressure induced uncertainties though the selection of measurement and reference wavelengths by matching individual spectral lines in the measurement and reference channels having similar spectral characteristics. The CoBRA method also has the advantage of providing a possibility to closely match weighting functions in the measurement (i.e. molecule of interest) and reference (i.e. oxygen.) bands holding potential for self-sufficient measurements of atmospheric molecular mixing ratios without external corrections for temperature and other atmospheric parameters. ${ }^{9}$

The key component of the CoBRA methodology is the temperature compensation throughout the entire range of total pressure variations and the weighting functions matching by properly selecting combinations of spectral lines and excitation wavelengths. In this paper we describe selected components of the validation methodology we employed to verify the temperature and total pressure sensitivity compensation potential of the Cross-Band Relative Absorption Technique (CoBRA) for the purpose of molecular mixing ratio measurements in the atmosphere. Results of the CoBRA temperature / total pressure sensitivity analysis in selected spectral bands for the molecules of $\mathrm{CO}_{2}$ and $\mathrm{CH}_{4}$ are presented including suitability comparisons of the A-Band and 1.26-1.27 micron band of oxygen for the CoBRA technique. Additional considerations related to the water vapor and aerosols interferences as well as the background correction techniques are discussed.

\section{CoBRA TECHNIQUE OVERVIEW AND VALIDATION ALGORITHM IMPLEMENTATION}

The implementation of the CoBRA technique is based on two components: (1) the selection of spectral lines with close-matching spectral parameters and (2) the proximity of half-width relative spectral distances from the center of the line to the location of the measurement wavelengths $\left(\Delta f_{m}\right.$ and $\left.\Delta f_{r}\right)$ as shown in Fig. 1

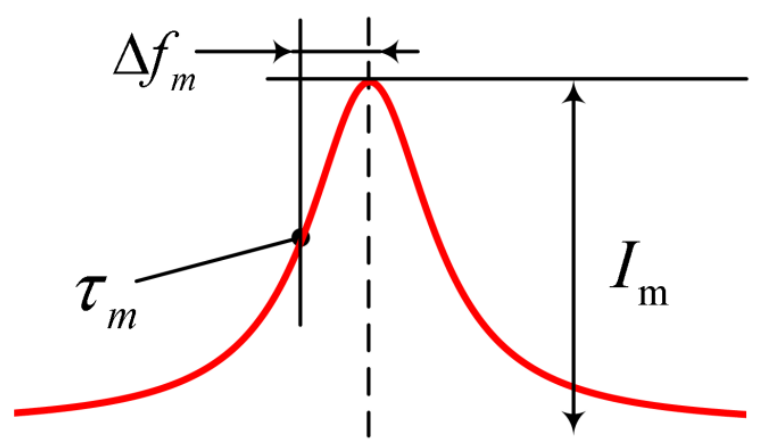

Measurement line

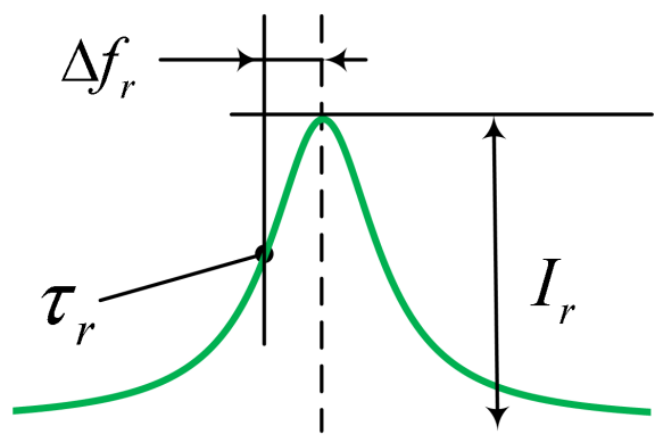

Reference line

Fig. 1 Illustration of the spectral line matching concept to achieve better temperature cancelation conditions using the cross-band relative absorption technique

The exact match of line intensities $\left(I_{m}\right.$ and $\left.I_{r}\right)$ is not required, however the proximity of line intensities is desirable due to limitations on the usable optical depth values suitable for the space lidar applications. ${ }^{10}$ These limits on the optimum optical depth impose restrictions on the available spectral line regions for cross-band line matching. Such limitation dictated by the line intensity induced constraints is illustrated further in this paper by performing the CoBRA analysis for the Methane $\left(\mathrm{CH}_{4}\right)$ molecule using the A-Band of oxygen.

The validation methodology for the CoBRA technique we have selected is based on the HITRAN database, Lineby-line radiative transfer model (LBLRTM), and the modern-era retrospective analysis for research and applications (MERRA). ${ }^{11-13}$ Our method employs pre-analysis of the MERRA dataset on a temperature / pressure grid to reduce the number of calculation required as described in our precious presentation. ${ }^{14}$ The selected temperature / pressure binning 
methodology for a span of year 2009 and all locations is further explained in Fig. 2. As can be seen, the original analysis of the "inst6_3d_ana_Nv" MERRA data was performed on a grid of 1 Kelvin x 1 mbar as shown in Fig 2a. The finer $1 \mathrm{mbar}$ bins where then merged together to correspond to the 72 tabulated layers of the MERRA dataset thus reducing the number of pressure level grids to 72 . These 72 bins were selected so that the tabulated MERRA pressure level values shown in Fig 2band 2c correspond to the centers of the pressure level bins formed. The reduction of the altitude (pressure) level bins was partially dictated by the need to reduce the number of calculations necessary to perform the complete temperature / pressure sensitivity analysis.
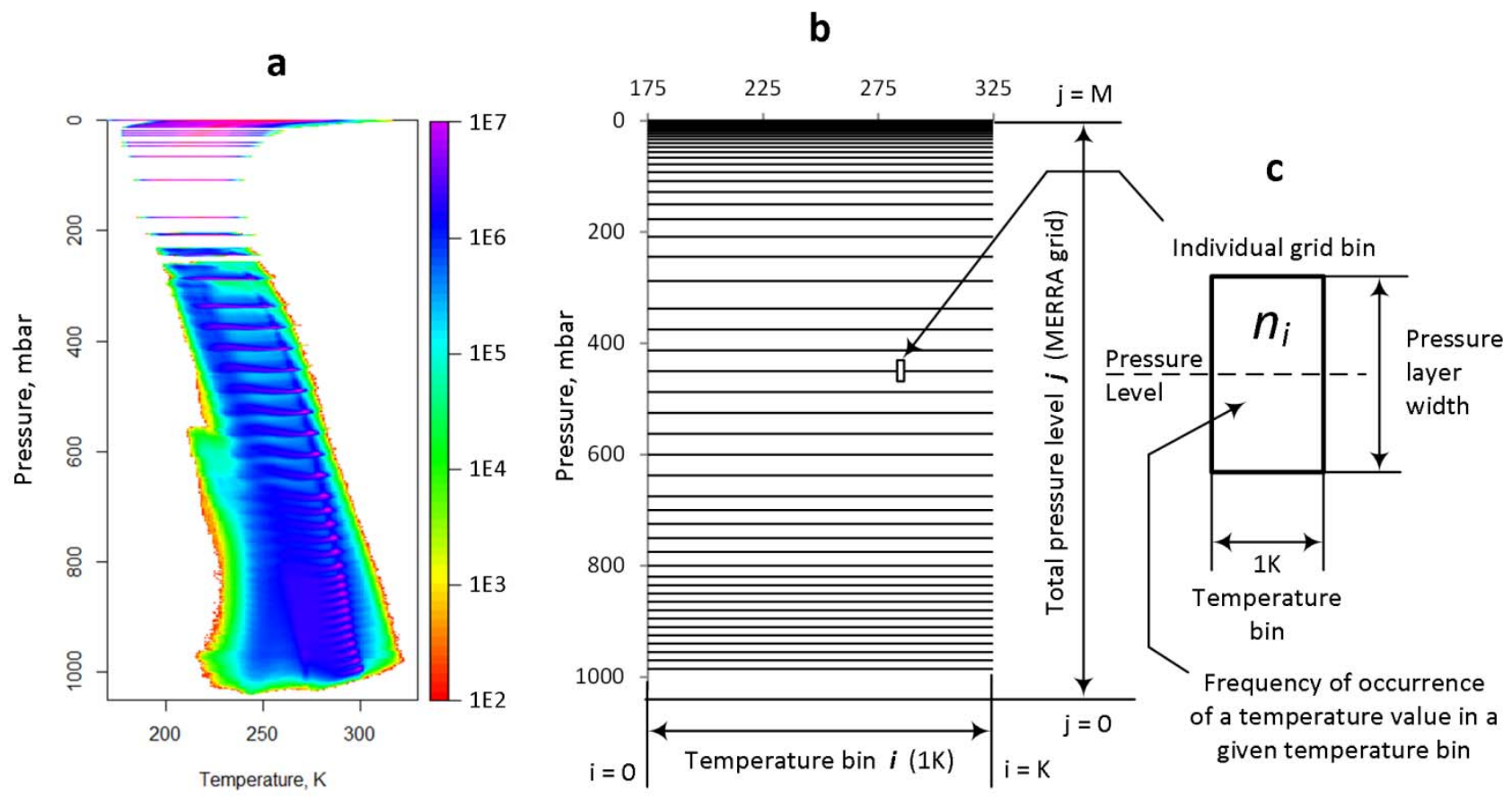

Fig 2 Results of the MERRA dataset temperature analysis on a $1 \mathrm{~K}$ x $1 \mathrm{mbar}$ grid with color coded frequency of occurrence of temperature /.total pressure combinations (a), binning methodology and indexing with MERRA tabulated pressure level grid of 72 layers applied to the analyzed MERRA data in (a), and the illustration of the individual bin selected dimensions (c).

After the initial MERRA dataset pre-analysis and the simulation approach involved the use of the LBLRTM program to obtain a set of transmission spectra at selected temperature and pressure combinations for subsequent calculation of the total temperature and pressure induced uncertainties. The description of our calculation methodology and analytical formulas used maybe found in our related publication and is further illustrated in Fig 3 shown. ${ }^{9}$ As can be seen, the atmospheric girded data is used to carry out transmission calculations for all available temperature combinations within each pressure level. The line-by-line calculations were performed by maintaining the path length of $1 \mathrm{~km}$ for each of the 72 calculated layers. Since the final calculation results are relative values, the actual layer thickness was kept equal to $1 \mathrm{~km}$ for calculation convenience. The transmission spectra are then used to calculate the variances due to changes in temperature within each layer for 3 different temperature analysis types: (1) Uncorrected, (2) 2 wavleength IPDA, and (3) 2-wavelength CoBRA. The first temperature sensitivity case called "uncorrected" is performed at separate wavelengths without using any off-line or reference channels results in the temperature-induced error estimates to be expected if a single measurement wavelength is used. The IPDA and CoBRA temperature estimate cases utilize one-offline and one reference wavelength respectively. As such, the analysis of the IPDA and CoBRA sensitivity involves considerations of all possible wavelength combinations under identical temperature and pressure conditions. The analytical formulas representing the variables in the Uncorrected, IPDA, and CoBRA temperature sensitivity analysis are presented in Eq. $1-3$ 


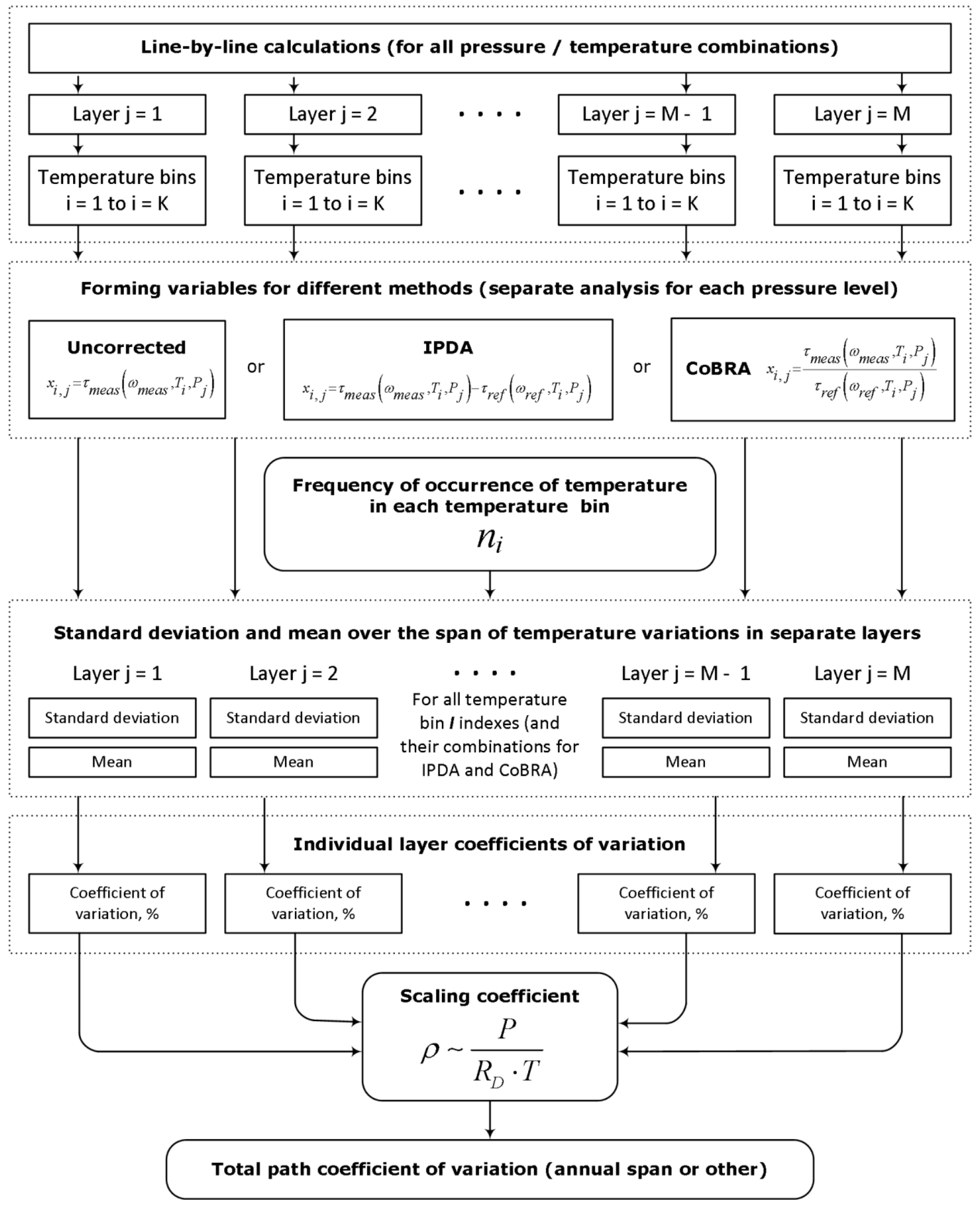

Fig. 3 Illustration of the temperature sensitivity calculation methodology including individual pressure layer analysis and combining of the individual layer uncertainty results into the total path temperature induced error. 


$$
\begin{gathered}
\text { Uncorrected: } x_{i, j}=\tau_{\text {meas }}\left(\omega_{\text {meas }}, T_{i}, P_{j}\right) \\
\text { IPDA: } x_{i, j}=\tau_{\text {meas }}\left(\omega_{\text {meas }}, T_{i}, P_{j}\right)-\tau_{\text {ref }}\left(\omega_{\text {ref }}, T_{i}, P_{j}\right) \\
\text { CoBRA: } x_{i, j}=\frac{\tau_{\text {meas }}\left(\omega_{\text {meas }}, T_{i}, P_{j}\right)}{\tau_{\text {ref }}\left(\omega_{\text {ref }}, T_{i}, P_{j}\right)}
\end{gathered}
$$

where $i$ - is the index of the temperature bin within a pressure layer $j$, Ti and $\mathrm{Pj}$ and the temperature and pressure corresponding to the temperature bin $i$ and a pressure level $j$ respectively, $\tau_{\text {meas }}$ and $\tau_{\text {ref }}$ are the optical depth values in the measurement and reference bands respectively at the measurement and reference wavelengths of $\omega_{\text {meas }}$ and $\omega_{\text {ref }}$

These variables are further used to calculate the variances and mean value for further determination of the relative coefficient of variation representative of the temperature induced error within each layer. The combining of the individual layer errors into the total path uncertainty is accomplished by scaling individual layer errors by the total molecular density at the corresponding pressure levels. We have selected a validation approach based on the calculation of variances as opposed to multivariate techniques such as PCA partly due to the ease of comparison with the results of experimental measurements.

The capability to calculate 3 different cases of temperature dependences (Uncorrected, IPDA, and CoBRA) enables cross-comparison of pure temperature sensitivity with that resulting from the application of IPDA and CoBRA methods. Pure temperature analysis for the alternative bands of $\mathrm{CO} 2$ and $\mathrm{O} 2$ considered for the ASCENDS mission applications have been reported in Ref. 15. The results of such comparison will be presented in the next section

\section{UNCORRECTED, IPDA AND COBRA ANAL YSIS FOR SELECTED LINES OF $\mathrm{CO}_{2}$}

We have used the calculation methodology described in section 2 to determine the temperature induced optical depth uncertainties as a function of wavelength for the $\mathrm{CO}_{2}$ in the $1.57 \mu \mathrm{m}$ band for the uncorrected, IPDA and CoBRA approaches. Other molecules and spectral bands were also investigated. Such analysis allowing comparison with the uncorrected optical depth uncertainties introduced due to temperature variations for different spectral lines and regions is useful as it provides quantitative reference information for evaluating the level of improvements in temperature induced uncertainties due to the introduction of an off-line wavelength (i.e. IPDA) or of the reference oxygen channel (CoBRA). Fig. 4 shows the comparison of the maximum and minimum achievable CoBRA technique accuracies in the measurement $\mathrm{CO} 2$ band of 1.57 micron referenced to the band of .oxygen in the 126-1.27 micron region. The point distribution plots displayed in Fig $4 \mathrm{c}$ and $4 \mathrm{e}$ are the maximum and minimum possible accuracies at as a function of measurement wavelength for all possible off-line combinations of reference wavelengths in the band of oxygen. For comparison the uncorrected coefficient of variation is plotted in Fig $4 \mathrm{~b}$ and $4 \mathrm{~d}$. As can be seen, the CoBRA method results in significant accuracy improvements compared to uncorrected uncertainties at the optimum wavelength combinations but may also result in increased errors exceeding the uncorrected values for the worst case selection of wavelength pairs. The "uncorrected" temperature sensitivity analysis shown in Fig $4 \mathrm{~b}$ and $4 \mathrm{~d}$ was carried out in the same way as that for the IPDA and CoBRA calculations with the exception that no off-line or reference wavelengths were used by performing single wavelength uncorrected temperature sensitivity calculations.

It should be noted that the distribution of the low temperature sensitive regions in the 1.57 micron band of $\mathrm{CO}_{2}$ shown in Fig $4 \mathrm{~b}$ and $4 \mathrm{~d}$ is in agreement with the temperature sensitivity analysis results for the 1.57 micron band 
previously reported by Menzies et. al. further supporting the correctness of the results obtained using our validation methodology . ${ }^{1}$
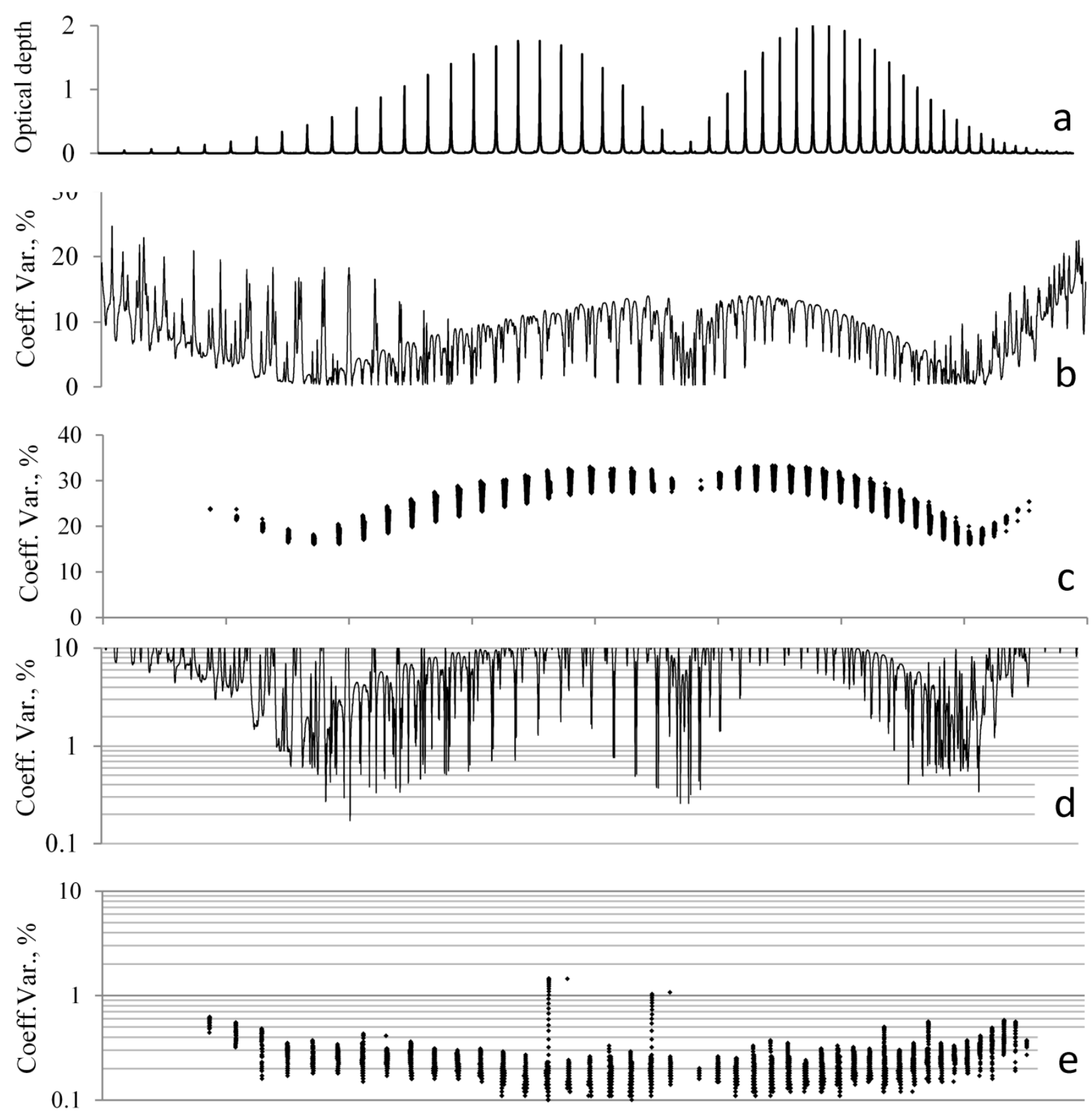

Fig 4 Comparison of uncorrected temperature induced uncertainties in the 1.57 micron band of CO2 (b and d) with the maximum (c) and minimum (e) achievable temperature induced uncertainties using the CoBRA method. Spectrum in (a) is a US Standard atmosphere calculated spectrum for an a vertical path of $80 \mathrm{~km}$. The results are for due to annual variations in atmospheric temperature and pressure for year 2009. 

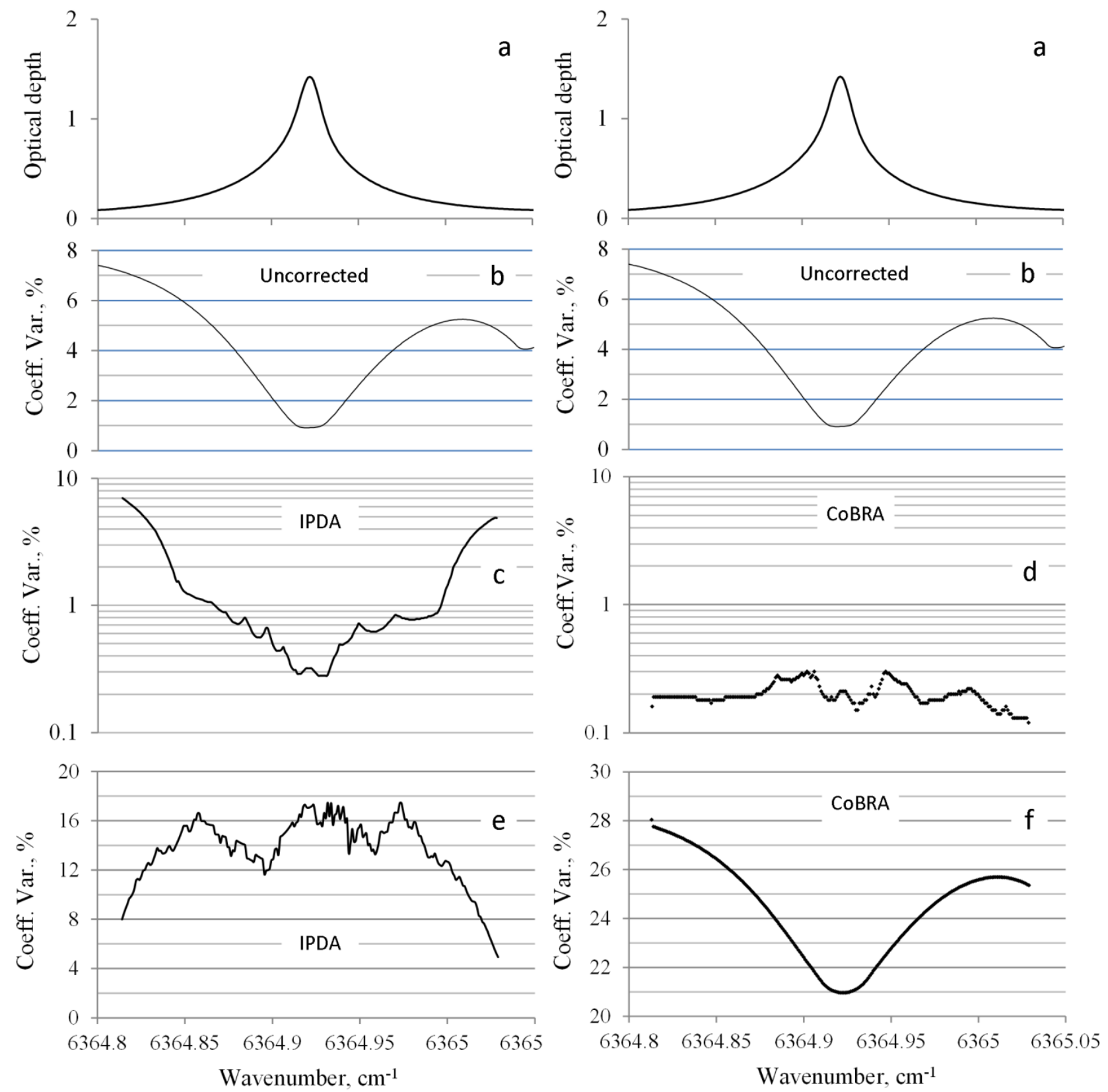

Fig 5 Comparison of maximum and minimum achievable temperature induced errors using IPDA (c, e) and CoBRA (d, f) techniques with the uncorrected temperature induced error levels (b) for a sample CO2 line in the $1.57 \mu \mathrm{m}$ band (a).

The comparison of the results displayed in Fig $4 \mathrm{~d}$ and $4 \mathrm{e}$ also shows that low temperature sensitivity levels may be achieved using the CoBRA approach even for spectral regions which exhibit the highest uncorrected temperature sensitivities. Error reductions for such regions drastically expand the span of possible excitation wavelengths. This result is particularly important from the stand point of additional optimizations such as those involving selection of wavelengths to reduce water vapor interferences.

We will now look into the comparison of the IPDA and Cobra performances by looking at a single $\mathrm{CO}_{2}$ absorption line as shown in Fig 5. As can be seen, Fig 5a represents the optical depth for a vertical path of 80km. Fig. 5b displays 
the total path uncorrected temperature induced uncertainties. Of importance is the observation that the temperature induced uncertainties increase as the wavelength is shifted away from the center of the line as shown in Fig $5 \mathrm{~b}$. The effect of the introduction of one off-line wavelength spanned through the $6300-6380 \mathrm{~cm}^{-1}$ region results in the minimum (Fig 5c) and maximum (Fig 5e) achievable error curves. The application of the CoBRA technique when the reference line is changed within the range of $7750-8000 \mathrm{~cm}-1$ is shown in Fig 5d and 5f, displaying the best and worst case error curves respectively. By comparing the figures one can see that the even though IPDA (Fig 5c) results in substantial accuracy improvements for the regions further away from the center of the line, only the CoBRA (Fig 5d) approach results in almost flat low error curve irrespective of the location with reference to the line center. This result is especially important from the stand point of the weighting function selection for improved surface sensitivity such as for the purpose of better capture of the sources and sinks of greenhouse gasses. It should be noted however, that improper selection of the measurement / reference wavelength combinations in CoBRA (Fig 5f) may result in the largest errors compared to the uncorrected (Fig 5b) and IPDA (Fig 5e) data.

\section{CoBRA ANALYSIS FOR SELECTED SPECTRAL LINES OF METHANE (CH4)}

Our CoBRA technique sensitivity analysis also involved other molecules such as $\mathrm{CH}_{4}$ and $\mathrm{N}_{2} \mathrm{O}$. The results of such calculations for the cross-band matching of $\mathrm{CH}_{4}$ in the 5830-6150 $\mathrm{cm}^{-1}$ spectral regions with the bands of oxygen in the 1.26-1.27 micron region sand the A-Band are presented in Fig 6. Fig 6a and 6b display the point distribution of the wavelength combinations resulting in the total path uncertainties of $0.3 \%$ or lower when matched with the spectral bands of oxygen shown in Fig 6d and 6e.
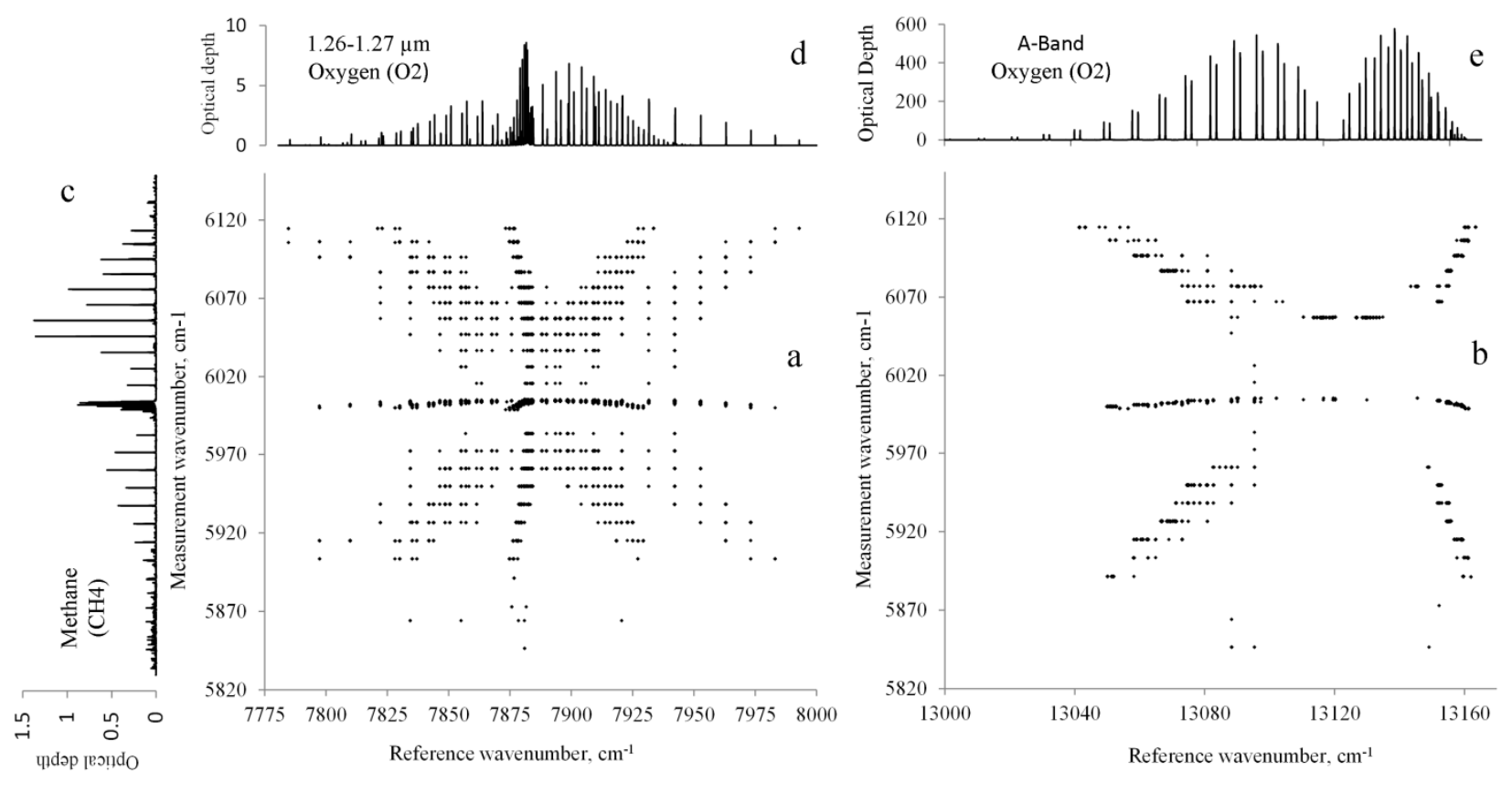

Fig 6 Comparison of CoBRA spectral lines matching $(a, b)$ point distributions with values of $0.3 \%$ or less for a band of $\mathrm{CH} 4$ (c) with the 1.26-1.27 $\mu \mathrm{m}(\mathrm{d})$ and the A-Band (e) of oxygen respectively.

As can be seen, the patterns observed is similar to that reported in our related study for the $1.57 \mu \mathrm{m}$ band of $\mathrm{CO}_{2}{ }^{9}{ }^{9}$ The calculation was performed by keeping the concentration of $\mathrm{CH}_{4}$ constant regardless of the altitude as this did not affect the results of the calculations due to low concentration of $\mathrm{CH}_{4}$ in the atmosphere and the proximity of the self- and 
air- broadening coefficients in the HITRAN database ${ }^{11}$ We could have also used an altitude concentration distribution $\mathrm{CH}_{4}$ profile in our calculations such as the US standard model to take into account the changes in the concentration of $\mathrm{CH}_{4}$ with altitude. However, since the concentration of these gasses is small in the atmosphere and the self- and airbroadening coefficients differ insignificantly to result in noticeable additional errors compared to those due changes in temperature profiles, it was decided to use the same concentration at all altitudes for convenience. Such choice was also dictated by the fact that our selected calculation technique relies on the determination of relative uncertainties and as such does not require calculation of the total optical depth values (except for the purpose of limiting the calculation spectral range to the favorable optical depth range of $\sim 0.1$ to 2 as described in Ref. 9 . In particular, the concentrations of the $\mathrm{CH} 4$ gas was maintained at the US standard surface values of $1.7 \mathrm{ppmv}$.

To further look at the best achievable accuracies for these 2 cases considered (6a and 6b) we have plotted (Fig. 7) the point distribution of the best and worst achievable uncertainties as a function of measurement wavelength in the CoBRA method for cross band matching of CH4 (Fig. 6c) with the 2 spectral bands of oxygen shown in (6d and 6e)
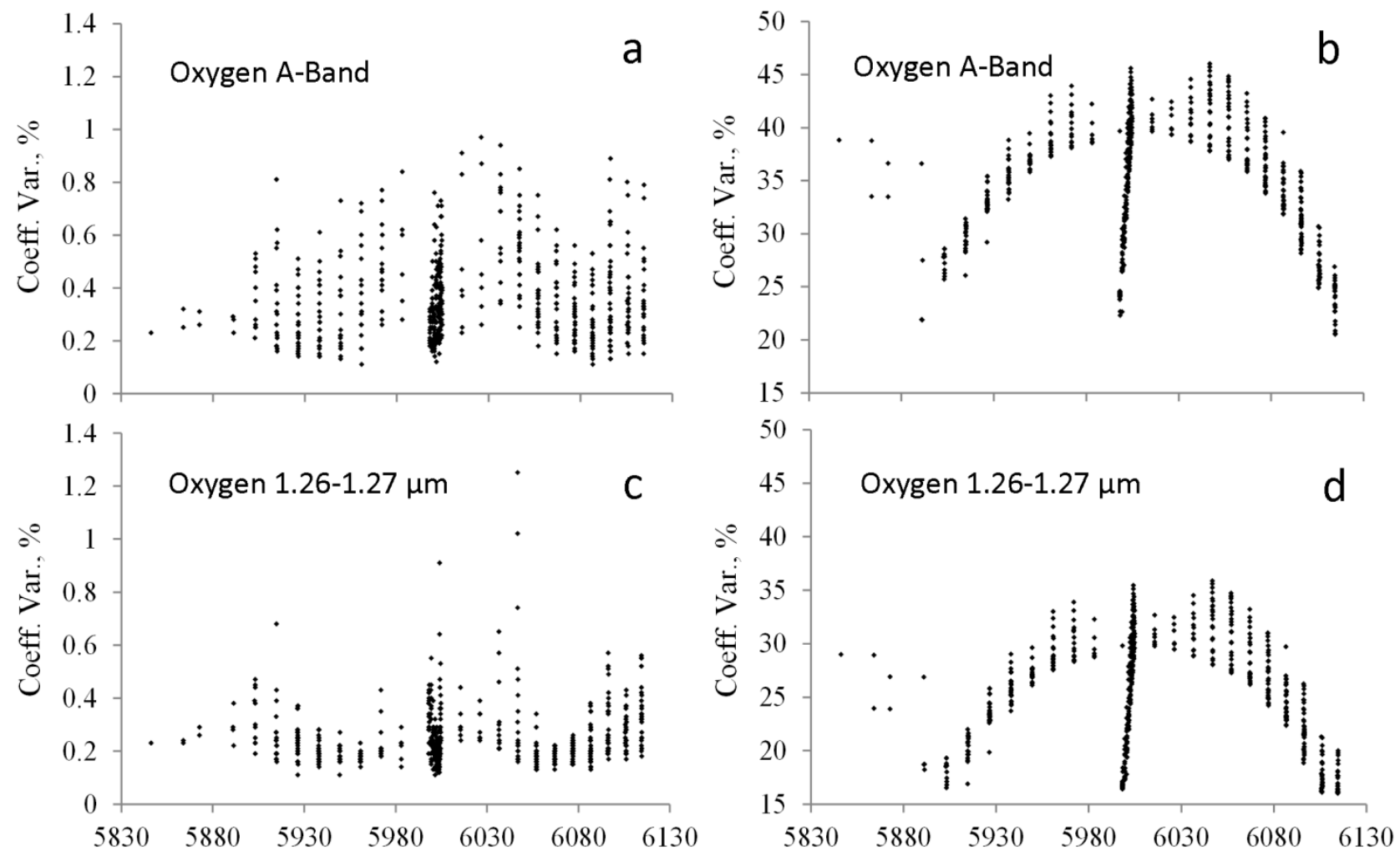

Fig 7 Comparison of the minimum and maximum (a, $\mathrm{c}$ and b, $\mathrm{d}$ respectively) calculated temperature induced errors for the CoBRA matching of $\mathrm{CH}_{4}\left(5830-6150 \mathrm{~cm}^{-1}\right.$ region) with the A-Band and $1.26-1.27 \mu \mathrm{m}$ band of oxygen.

As can be seen, we are getting the lowest achievable temperature induced uncertainty of $0.1 \%$ when matching of CH4 spectra is performed. The same best performance limit of $0.1 \%$ for the temperature induced uncertainties was observed for the CoBRA matching of $\mathrm{CO}_{2}$ molecule in our related study. ${ }^{9}$ Due to the accuracy limitations of the Voigt profile, computations using advanced lineshape models and additional attenuation components such as collision induced absorption and line mixing are further needed to further establish the best achievable temperature insensitivity limits. The results presented in Fig 7 with different reference spectral bands of oxygen used show comparable best achievable performance (Fig 7a and 7c) for both of the alternative $\mathrm{O}_{2}$ spectral bands used. As a reminder we will point out that the point distribution plots shown in Fig 7 were obtained by scanning the entire set of analyzed wavelength combinations to 
preserve only the best and worst cases at each of the measurement wavelengths which the reference wavelength is allowed to span the entire range of wavelengths shown in Fig $6 \mathrm{~d}$ and $6 \mathrm{e}$ respectively.

To establish if the strong oxygen lines in the A-Band may be used for the CoBRA wavelengths pairs we have expanded a sub-region of Fig 7a as shown in Fig 8a referenced to the total optical depth spectrum of $\mathrm{O}_{2}$ in the corresponding spectral range of $13070-13085 \mathrm{~cm}^{-1}$ shown in Fig $8 \mathrm{~b}$.

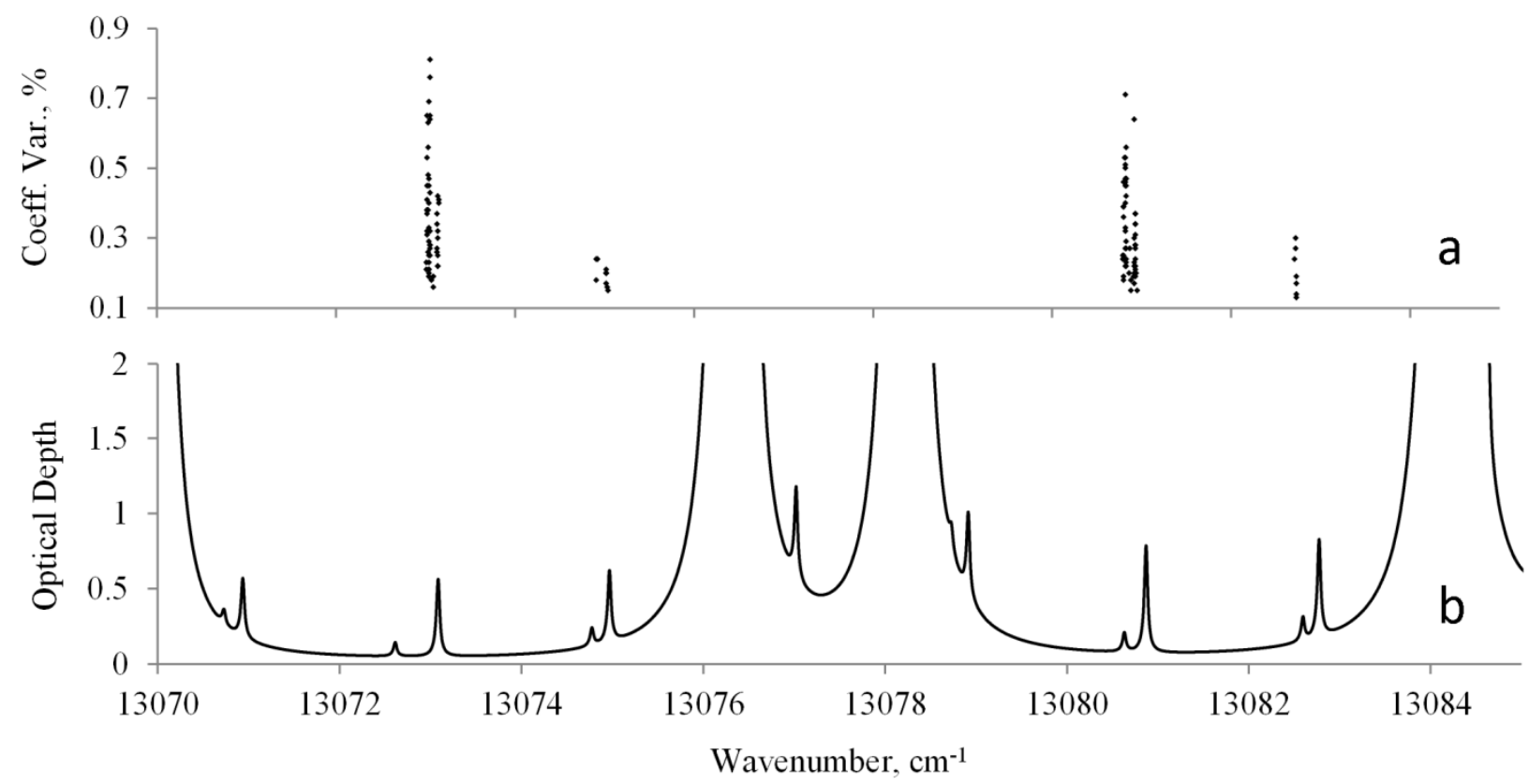

Fig 8 Point distribution of the minimum achievable temperature induced errors (a) and the corresponding reference oxygen spectrum (b) for the CoBRA analysis of $\mathrm{CH}_{4}\left(5830-6150 \mathrm{~cm}^{-1}\right.$ spectral region)

As can be seen from Fig 8, CoBRA wavelength matching could not be performed at the wings of the strong absorption lines, instead the best matching is achieved for the week spectral lines more remote from the overly intense ones. These results suggest that the A-Band has limited application in the CoBRA methodology if combined with lower intensity measurement bands compared to the $1.26-1.27$ micron band of oxygen due to significant differences in the line intensities between the measurement spectral bands and the A-Band strong oxygen lines. Our additional studies indicate that the A-Band is well suited for CoBRA applications using more intense measurement bands such as the 2.05 $\mu \mathrm{m}$ band of $\mathrm{CO}_{2}{ }^{16}$

\section{FURTHER ANALYSIS}

Our further CoBRA technique analysis not presented in this paper includes considerations of the weighting functions matching, water vapor interferences, aerosols and presence of multiple interference species. Initial considerations of some of these questions have been reported in our related paper. ${ }^{9}$ These further complete results will be reported in separate publications and presentations. It should be pointed out that other molecules well mixed in the atmosphere such as $\mathrm{N}_{2}$ may be used for the reference band measurements using the CoBRA technique with comparable achievable accuracies expected. 


\section{PROJECTED OPEN SOURCE SOFTWARE IMPLEMENTATION OF CoBRA}

The methodology we have introduced for the quantitative estimates of temperature / pressure induced uncertainties is suitable for automated analysis such as using desktop computers and is currently being further improved to be incorporated into the modeling framework for space and airborne lidar sensitivity simulations and optimizations under development by our group.

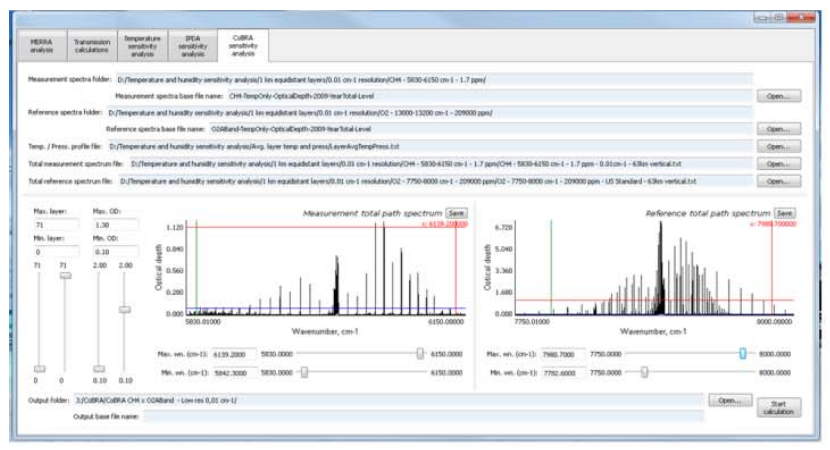

Temperature sensitivity, IPDA and CoBRA analysis

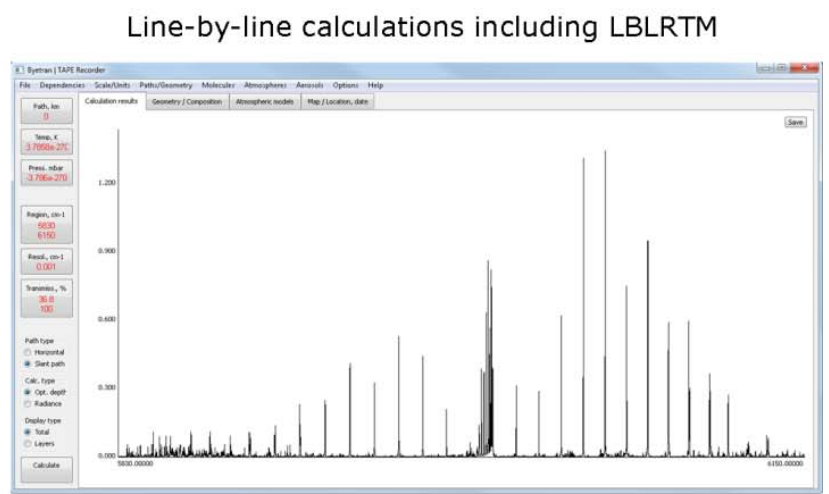

Fig 9 GUI screens of selected modeling programs being developed for spectral and lidar optimizations.

The GUI print screens of selected optimization software under development are presented in Fig 9. Once the calculation methodology is fully developed and refined it is in our plans to make the optimization modeling programs widely available.

\section{CONCLUSIONS}

A new approach has been proposed called cross-band relative absorption suitable for improved precision of molecular mixing ratio measurements. We have used a data reduction approach and a number of experimental and theoretical datasets to illustrate that significant reduction in temperature sensitivities is achievable by proper combination of measurements spectral lines and excitation wavelengths with application to the determination of mixing ratios of various gasses including atmospheric constituents. It is shown that the 1.26-1.27 micron band is better suited for the CoBRA implementation that the A-Band of oxygen. Experimental measurements are necessary to completely verify the CoBRA methodology and establish realistic achievable performance improvements.

It should be understood that the CoBRA technique is not a substitute of the existing IPDA approaches but rather an additional method which in combination with the current IPDA methodologies leads to the reduction of temperature induced sensitivities and thus expands the range of measurement wavelengths which may be selected for further water vapor and other interference reductions as well as for improved weighting functions selection. .

Additionally, the modeling algorithms implemented are a good fit for a desktop computer analytical application making it possible to implement a set of useful analytical software programs for wavelength selection optimizations including IPDA and CoBRA techniques.

\section{ACKNOWLEDGEMENTS}

The authors acknowledge the support from the Earth Science Technology Office (ESTO) and the NASA Postdoctoral Program (NPP) administered by Oak Ridge Associated Universities (ORAU). MERRA data used in this 
study have been provided by the Global Modeling and Assimilation Office (GMAO) at NASA Goddard Space Flight Center through the NASA GES DISC online archive.

\section{REFERENCES}

[1] Robert T. Menzies, and David M. Tratt, "Differential laser absorption spectrometry for global profiling of tropospheric carbon dioxide: selection of optimum sounding frequencies for high-precision measurements", Applied Optics, Vol 42, No. 33, pp. 6569-6577, (2003)

[2] Jerome Caron, Yannig Durand, "Operating wavelengths optimization for a spaceborne lidar measuring atmospheric $\mathrm{CO}_{2}$ ”, Appl. Opt. 48, 5413-5422 (2009)

[3] G. Ehret, C. Kiemle, M. Wirth, A. Amediek, A. Fix, S. Houweling,",Space-borne remote sensing of $\mathrm{CO}_{2}, \mathrm{CH}_{4}$, and $\mathrm{N}_{2} \mathrm{O}$ by integrated path differential absorption lidar: sensitivity analysis", Appl. Phys. B, 90, 593-608 (2008).

[4] C. Kiemle, M. Quatrevalet, G. Ehret, A. Amediek, A. Fix, and M. Wirth, "Sensitivity studies for a space-based methane lidar mission", Atm. Meas. Tech, Disc., 4, 3545-3592, (2011)

[5] “Active Sensing of CO2 Emissions over Nights, Days, and Seasons (ASCENDS) Mission”, NASA Science Definition and Planning Workshop, Report, Univ. of Michigan, Ann Arbor, MI, 23-25 July, 2008

[6] C. Stephan ; M. Alpers ; B. Millet ; G. Ehret ; P. Flamant ; C. Deniel; MERLIN: a space-based methane monitor. Proc. SPIE 8159, Lidar Remote Sensing for Environmental Monitoring XII, 815908 (September 13, 2011); doi: $10.1117 / 12.896589$

[7] Jérôme Caron ; Yannig Durand ; Jean-Loup Bezy ; Roland Meynart; Performance modeling for A-SCOPE: a spaceborne lidar measuring atmospheric CO2. Proc. SPIE 7479, Lidar Technologies, Techniques, and Measurements for Atmospheric Remote Sensing V, 74790E (October 09, 2009); doi:10.1117/12.830364

[8] R. T. Menzies and M. T. Chahine, "Remote Atmospheric Sensing with an Airborne Laser Absorption Spectrometer," Appl. Opt. 13, 2840-2849 (1974)

[9] N. Prasad, D. Pliutau, "Cross-band relative absorption technique for the measurement of molecular mixing ratios", (submitted to Optics Express)

[10] Ellis E. Remsberg and Larry L. Gordley, "Analysis of differential absorption lidar from the space shuttle," Appl. Opt. 17, 624-630 (1978).

[11] S. Rothman, Iouli Gordon et al.,’The HITRAN 2008 molecular spectroscopic database”, JQSRT, 110, 533-572 (2009).

[12] S.A. Clough, M.W. Shephard, E.J. Mlawer, J.S. Delamere, M.J. Iacono, K. Cady-Pereira, S. Boukabara, P.D. Brown, "Atmospheric radiative transfer modeling: a summary of the AER codes", JQSRT, 91, 233-244 (2005).

[13] Rienecker, M.M., M.J. Suarez, R. Gelaro, R. Todling, J. Bacmeister, E. Liu, M.G. Bosilovich, S.D. Schubert, L. Takacs, G.-K. Kim, S. Bloom, J. Chen, D. Collins, A. Conaty, A. da Silva, et al., ” MERRA - NASA's Modern-Era Retrospective Analysis for Research and Applications", J. Climate, 24, 3624-3648, (2011).

[14] D. Pliutau, N. Prasad, "Simulation studies for comparative evaluation of alternative spectral regions for the sensing of $\mathrm{CO}_{2}$ and $\mathrm{O}_{2}$ suitable for the ASCENDS Mission", Proc. SPIE 8513, 851309 (2012).

[15] D. Pliutau, N. Prasad, "Comparative analysis of alternative spectral bands of $\mathrm{CO}_{2}$ and $\mathrm{O}_{2}$ 'for the sensing of $\mathrm{CO}_{2}$ mixing ratios", accepted to SPIE Defense Security and sensing 2013, Baltimore, MD, 29 April - 3 May, 2013

[16] D. Pliutau, N. Prasad, "Cross-band Relative Absorption Technique for Molecular Mixing Ratio Determination", submitted to CLEO 2013, San Jose CA, 9 - 14 June, 2013 